\title{
Assessment of serum Talin-1 in liver cirrhosis and hepatocellular carcinoma
}

\author{
El Said H. Ibrahim ${ }^{1}$, Hend Naguib ${ }^{1}$, Doaa M. Emara², Eman T. El Sayed ${ }^{3}$ and Marwa M. R. Tawfik ${ }^{1,4^{*}}$ (D)
}

\begin{abstract}
Background: Early detection of hepatocellular carcinoma (HCC) is crucial for improving the survival rate for patients. Talin-1 is first identified as a cytoskeleton protein that is required for cell adhesion and motility and plays a role in tumor migration and metastasis. In the present work, we aimed to study the possible role of Talin 1 compared to alpha fetoprotein (AFP) in the diagnosis and prognosis of HCC.

Methods: To achieve this goal, serum levels of Talin-1 were measured using enzyme-linked immunosorbent assay (ELISA) in 90 patients divided into four groups. Group I: 30 patients with early HCC. Group II: 30 patients with late HCC according to Modified Barcelona-Clinic Liver Cancer (BCLC). Group III: 15 patients with liver cirrhosis, and group IV: 15 healthy controls. Receiver operating characteristics (ROC) curve analysis was used to create a predictive model for Talin-1 relative to AFP in HCC diagnosis.

Results: It was found that serum Talin-1 in HCC patients was significantly higher compared to its level in cirrhotic patients and the healthy control group. Talin-1 was superior to AFP regarding sensitivity, specificity, positive, and negative predictive value in the diagnosis of HCC. We also found a significant positive correlation between serum Talin-1 and the degree of tumor burden of HCC (BCLC staging), tumor size, and vascular invasion.
\end{abstract}

Conclusion: Talin-1 holds a promise as a potential marker for HCC diagnosis and prognosis.

\section{Introduction}

Hepatocellular carcinoma (HCC) is one of the most prevalent cancers worldwide [1]. About 10.9 million new cases of cancer are diagnosed annually, thus representing an international public health problem [2]. It is ranked as the sixth most common cancer. In the absence of surveillance and early detection programs, HCC presents late, at which curative therapy can seldom be applied safely and when feasible management is not effective. In Egypt, there is an increasing incidence of $\mathrm{HCC}$, representing the leading cause of death among patients with chronic liver disease [3]. In the USA, about 35,660 new cases were diagnosed in 2015 [4].

\footnotetext{
*Correspondence: mrtawfik@pnu.edu.sa; drmarwareda@hotmail.com ${ }^{4}$ Clinical Science Department, College of Medicine, Princess Nourah bint Abdulrahman University, Riyadh, KSA

Full list of author information is available at the end of the article
}

Liver cirrhosis is the main risk factor for $\mathrm{HCC}$ and represents about $80 \%$. The common causes of liver cirrhosis are chronic hepatitis B virus (HBV) infection or chronic hepatitis $\mathrm{C}$ virus (HCV) infection, alcoholics, non-alcoholic liver disease, aflatoxin exposure, obesity, and iron overload [5]. Irrespective of the etiology of cirrhosis, the annual incidence of progression from chronic hepatitis to cirrhosis to HCC is 3-7\% [6]. Early diagnosis of HCC is crucial and allows the physician to apply curative and effective treatment, thus improving the survival rate. Therefore, achieving successful screening tool to be applied to patients with chronic liver disease who are at risk for $\mathrm{HCC}$ is critically important and challenging nowadays [7].

Serum AFP level is a useful tumor marker for the detection and monitoring of HCC. AFP is not always specific for HCC; it can be elevated in other carcinomas like gastric cancer and lung cancer [8], and liver disease other than HCC, such as hepatitis or cirrhosis. Also, AFP is not 
secreted in all cases of $\mathrm{HCC}$, so the false-negative rate may be as high as $40 \%$ for patients with early-stage HCC. Its poor sensitivity renders it unsatisfactory for this purpose and suggests an urgent need for novel biomarkers for early-stage HCC detection [9].

Integrins are mediators of cell-extracellular matrix interaction, not only provide physical links with the cytoskeleton but also transduce signals from the ECM to the cell, mandatory for several cellular processes including migration, proliferation, and survival. One of the key proteins required for the assembly of cell-matrix adhesions is the adaptor protein Talin, which mediates the interaction between the actin cytoskeleton and integrins [10]. Talin-1 is expressed mainly in the kidney, liver, spleen, stomach, lung, and vascular smooth muscle [11]. Talin expression also is highly associated with endometrioid carcinoma and prostate cancer [12]. Recently Talin-1 was identified by differential tissue proteome as a novel molecular marker for HCC progression [13]. Talin is (270 $\mathrm{kDa}, 2541$ amino acids), which is required to maintain cell spreading, cell migration, and focal adhesion formation [14]. This work aimed to study the role of serum Talin-1 in the diagnosis of HCC in comparison to AFP as regards negative and positive predictive values and to correlate it with the tumor characteristics as regards size and vascular invasion.

\section{Materials and methods}

This study was conducted on 90 patients who were admitted to Hepatobiliary Unit or followed in the hepatobiliary outpatients' clinic, Alexandria Main University Hospital, Alexandria, Egypt. They were divided into four groups. Group I: 30 patients with early HCC with tumor characteristic of stages 0, A, B (very early, early, and intermediate) according to modified Barcelona-Clinic Liver Cancer (BCLC). Group II: 30 patients with late HCC with tumor characteristic of stages $C$, D according to modified BCLC (advanced and end-stage), Group III: 15 patients with $\mathrm{HCV}$-related liver cirrhosis, and group IV: 15 age and sex-matched healthy subjects with no evidence of liver disease were included as a control group to obtain the normal range of biochemical assays. All participants were evaluated clinically as regards manifestations of the advanced liver disease, liver, spleen size, and presence or absence of ascites. HCC staging was done according to modified BCLC including tumor features, liver functions (according to Child-Pugh score), and patient's performance status. Routine investigations were done including complete blood picture, blood urea level, serum creatinine, and liver test profile. Serological viral testing was done including $\mathrm{HCV}$ antibodies, hepatitis B surface antigen, and total hepatitis B core antibodies. Also, autoimmune markers and tumor markers including prostatic-specific antigen and serum AFP were done. All participants were subjected to measurement of serum levels of Talin-1 using the ELIZA technique. Abdominal ultrasonography was done for assessment of the liver, spleen, portal circulation, and ascites. Diagnosis of HCC was obtained by multiphasic contrast-enhanced computer tomography $(\mathrm{CT})$ or dynamic contrast-enhanced magnetic resonance imaging (MRI) to assess $\mathrm{HCC}$ imaging hallmarks, size, number of the lesion(s), and vascular involvement and thrombosis.

\section{Principle of the serum Talin 1 assay by using standardized ELISA kit}

This assay employed the quantitative sandwich enzyme immunoassay technique. Antibody specific for TLN1 had been pre-coated onto a microplate. Standards and samples were pipetted into the wells, and any TLN1 present was bound by the immobilized antibody. After removing any unbound substances, a biotin-conjugated antibody specific for TLN1 was added to the wells. After washing, avidin-conjugated horseradish peroxidase (HRP) was added to the wells. Following a wash to remove any unbound avidin-enzyme reagent, a substrate solution was added to the wells and color develops in proportion to the amount of TLN1 bound in the initial step. The color development was stopped, and the intensity of the color was measured.

Patients with rheumatoid arthritis, alcohol abuse, autoimmune liver diseases, and active infections or prostatic carcinoma were excluded from our study. Also, absolute contraindication to multiphasic contrast-enhanced CT or refusing triphasic $\mathrm{CT}$ imaging was considered. The study protocol was approved by the Research Review Committee of the Alexandria Faculty of Medicine and was conformed to the 1975 Declaration of Helsinki. Informed consent was obtained from each subject included in the study.

\section{Statistical analysis}

Were performed using data fed to the computer using SPSS software. Qualitative data were described using number and percent. Quantitative data were described using range, mean, standard deviation, and median. The significance of the results was judged at the $5 \%$ level. The sensitivity and specificity of serum TALIN 1 in patients and controls were assessed by plotting (ROC) curve and determining its cut-off values.

\section{Results}

The present study was performed on 90 individuals who were classified into four groups, Group I, II, and III included patients with early HCC, late HCC, and $\mathrm{HCV}$-related liver cirrhosis respectively, while Group 
IV included healthy control. The mean age was $54.77 \pm$ $9.70,56.13 \pm 6.77,53.87 \pm 8.41$, and $49.93 \pm 11.0$ in the groups I, II, III, and IV, respectively; there was no significant difference between the studied groups $(p=0.177)$. Regarding sex, the male gender was more dominant in all studied groups than female, but with no significant difference $(p=0.07)$. Regarding the severity of liver disease, there was a significant difference between the three diseased groups, where most early HCC patients 21 (70\%) and cirrhotic patients $11(73.3 \%)$ were found to have B CHILD score, while most of late HCC patients 16 (53.3\%) had score C. According to the modified BCLC staging system for HCC, there was a significant difference between the two groups of HCC patients $\left(p^{<} 0.001\right)$, class B was more common 20 (66.7\%) in early HCC patients, while class D was more common 17 (56.7\%) in late HCC patients (Table 1).

The laboratory investigations for the studied groups are shown in Table 1. All investigated parameters were significantly differed between the four studied groups except for ALT. A sharp significant $(p<0.001)$ increase in the mean of AFP level was found in late HCC patients. Serum Talin-1 was significantly higher in HCC groups in comparison to other groups $(p<0.001)$. Also, there was a significant increase in the mean of S. Talin-1level in late HCC patients than in other groups ( $\left.p^{<} 0.001\right)$.

The receiver operating characteristics (ROC) curve was established to find out the sensitivity, specificity, positive predictive value (PPV), negative predictive value (NPV), and accuracy of both AFP and Talin-1 to diagnose HCC; the area under the curve (AUC) for S. Talin-1 is larger (0.997) than that of AFP (0.792). At cut-off $4.3 \mathrm{ng} / \mathrm{ml}$ for S. Talin gives a sensitivity of $95 \%$ and specificity of $93.3 \%$ with PPV and NPP equal $98.3 \%$ and $82.4 \%$, respectively, and accuracy of $94.7 \%$, while at cut-off $200 \mathrm{ng} / \mathrm{ml}$ for AFP the sensitivity is $25 \%$, specificity is $100 \%$ with PPV and NPV of $100 \%$ and $25 \%$, respectively, and accuracy of $40 \%$. At cut-off $9.1 \mathrm{ng} / \mathrm{ml}$ for AFP, the sensitivity, specificity, PPV, NPV, and accuracy resulted equal $66.7 \%, 93.3 \%$, $97.6 \%, 41.2 \%$, and $72 \%$, respectively (Fig. 1, Table 2 ).

There were no significant correlations between serum levels of Talin-1 and different CHILD scores $(r=0.051$, $p=0.077)$. There was a significant correlation between the level of serum Talin-1 and BCLC staging ( $p \leq 0.001$, $r=0.817$ ). Also, serum Talin-1 levels were significantly higher in patients with vascular invasion compared

Table 1 Comparison between the different studied groups according to demographic data, severity of liver disease, and the mean of investigated parameters and s. Talin-1 level

\begin{tabular}{|c|c|c|c|c|c|c|}
\hline & Early HCC $(n=30)$ & Late HCC $(n=30)$ & Cirrhotic $(n=15)$ & Control $(n=15)$ & Test of Sig. & $p$ \\
\hline Age (years) Mean $\pm S D$ & $54.77 \pm 9.70$ & $56.13 \pm 6.77$ & $53.87 \pm 8.41$ & $49.93 \pm 11.0$ & $F=1.683$ & 0.177 \\
\hline Sex & No. $(\%)$ & No. $(\%)$ & No. $(\%)$ & No. (\%) & & \\
\hline Male & $24(80)$ & $26(86.7)$ & $10(66.7)$ & $8(53.3)$ & $x^{2}=6.706$ & ${ }^{\mathrm{MC}} p=0.076$ \\
\hline Female & $6(20)$ & $4(13.3)$ & $5(33.3)$ & $7(46.7)$ & & \\
\hline \multicolumn{7}{|l|}{ CHILD score } \\
\hline A & $8(26.7)$ & $6(20.0)$ & $0(0.0)$ & & $x^{2}=26.030^{*}$ & ${ }^{M C} C_{p<0.001^{*}}$ \\
\hline B & $21(70.0)$ & $8(26.7)$ & $11(73.3)$ & & & \\
\hline C & $1(3.3)$ & $16(53.3)$ & $4(26.7)$ & & & \\
\hline \multicolumn{7}{|l|}{ BCLC score } \\
\hline A & $10(33.3)$ & 0 & & & $x^{2}=60.000$ & ${ }^{\mathrm{MC}} p<0.001^{*}$ \\
\hline B & $20(66.7)$ & 0 & & & & \\
\hline C & 0 & $13(43.3)$ & & & & \\
\hline $\mathrm{D}$ & 0 & $17(56.7)$ & & & & \\
\hline \multicolumn{7}{|l|}{ Lab Investigations } \\
\hline $\mathrm{Hb}(\mathrm{g} / \mathrm{dl})$ & $11.43 \pm 1.80$ & $11.44 \pm 1.88$ & $10.57 \pm 1.78$ & $13.73 \pm 1.21$ & $F=9.380^{*}$ & $<0.001^{*}$ \\
\hline WBCs $\left(10^{3}\right)$ & $5.45 \pm 2.82$ & $7.69 \pm 4.13$ & $5.57 \pm 2.80$ & $7.97 \pm 1.62$ & ${ }^{k W} X^{2}=13.312^{*}$ & $0.004^{*}$ \\
\hline Platelets $\left(10^{3}\right)$ & $111.43 \pm 77.54$ & $130.70 \pm 93.60$ & $87.20 \pm 53.46$ & $317.0 \pm 67.35$ & ${ }^{k W} X^{2}=32.619^{*}$ & $<0.001^{*}$ \\
\hline T. Bilirubin (mg/dl) & $1.55 \pm 1.08$ & $4.92 \pm 6.65$ & $2.39 \pm 3.05$ & $0.49 \pm 0.25$ & ${ }^{K W} X^{2}=39.160^{*}$ & $<0.001^{*}$ \\
\hline INR & $1.40 \pm 0.22$ & $1.67 \pm 0.40$ & $1.54 \pm 0.36$ & & $F=4.870^{*}$ & 0.01 \\
\hline S. Albumin (g/dl) & $2.81 \pm 0.60$ & $2.53 \pm 0.91$ & $2.40 \pm 0.59$ & $4.35 \pm 0.40$ & $F=27.315^{*}$ & $<0.001^{*}$ \\
\hline $\operatorname{AFP}(\mathrm{ng} / \mathrm{ml})$ & $693.9 \pm 2216.8$ & $4139.9 \pm 21178.5$ & $9.89 \pm 9.27$ & $6.02 \pm 2.24$ & ${ }^{K W} X^{2}=16.476^{*}$ & $0.001^{*}$ \\
\hline S. Talin-1 (ng/ml) & $6.77 \pm 1.72$ & $10.63 \pm 2.13$ & $4.19 \pm 1.45$ & $1.42 \pm 1.37$ & $F=102.437^{*}$ & $<0.001^{*}$ \\
\hline $\mathbf{p}_{\text {Con }}$ & $<0.001^{*}$ & $<0.001^{*}$ & $<0.001^{*}$ & & & \\
\hline
\end{tabular}




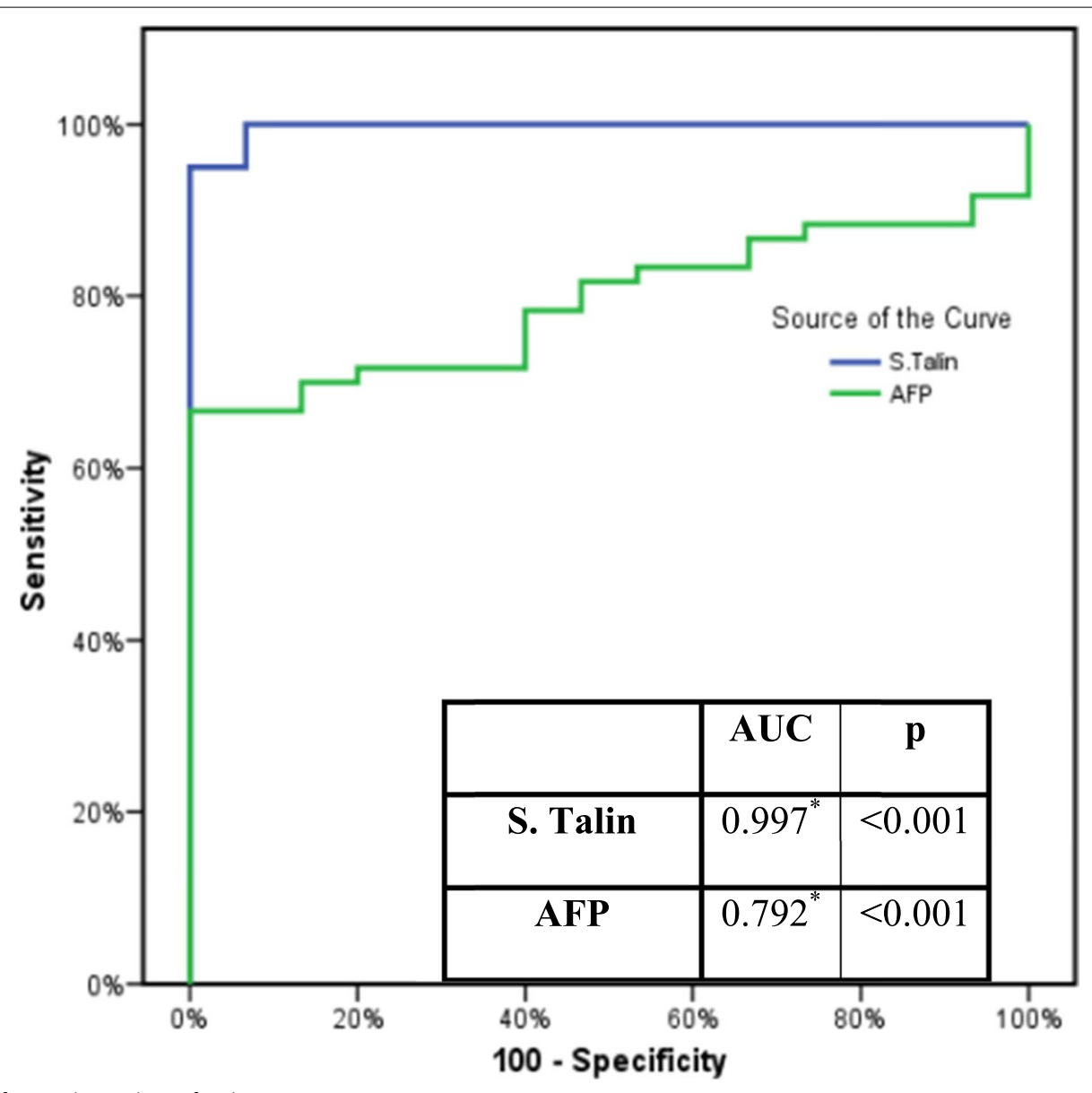

Fig. 1 ROC curve for S. Talin and AFP for diagnosing HCC patients

Table 2 Agreement (sensitivity, specificity, and accuracy) for s. Talin-1 and AFP with control and HCC groups

\begin{tabular}{|c|c|c|c|c|c|c|c|c|c|}
\hline Variables & Cut-off value & Control & $\mathrm{HCC}$ & Sensitivity & Specificity & PPV & NPV & Accuracy & $p$-value \\
\hline \multirow[t]{2}{*}{ S. Talin-1 } & $\leq 4.3$ & 14 & 3 & 95.0 & 93.3 & 98.3 & 82.4 & 94.7 & $<0.001$ \\
\hline & $>4.3$ & 1 & 57 & & & & & & \\
\hline \multirow[t]{2}{*}{ AFP } & $\leq 200$ & 15 & 45 & 25.0 & 100 & 100 & 25.0 & 40.0 & $<0.001$ \\
\hline & $>200$ & 0 & 15 & & & & & & \\
\hline \multirow[t]{2}{*}{ AFP } & $\leq 9.1$ & 14 & 20 & 66.7 & 93.3 & 97.6 & 41.2 & 72.0 & \\
\hline & $>9.1$ & 1 & 40 & & & & & & \\
\hline
\end{tabular}

to patients without vascular invasion in $\mathrm{CT}$ imaging $(p=0.001)$ (Fig. 2, Table 3). Moreover, there was a significant positive correlation between serum Talin-1 levels and tumor size $(r=0.267, p=0.040)$ (Table 3 ).

\section{Discussion}

Given that most $\mathrm{HCC}$ patients present with advanced disease, there is a pressing need for an effective biomarker that detects the presence and early stages of
$\mathrm{HCC}$ at a better capacity than AFP. The identification of a novel biomarker for $\mathrm{HCC}$ that can overcome these limitations may improve surveillance efforts and clinical outcomes. Ideal tumor biomarkers should possess high specificity and sensitivity not to be detected in premalignant liver disease. It should be easily accessed, easily measurable, minimally invasive, inexpensive, accurate, and acceptable to patients and physicians [15]. It has been found in the present study that serum 


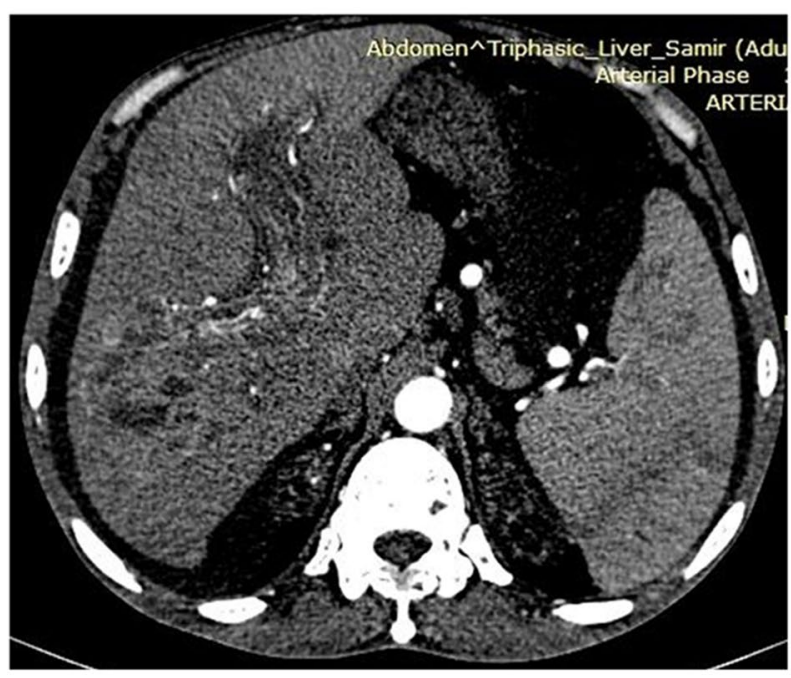

a

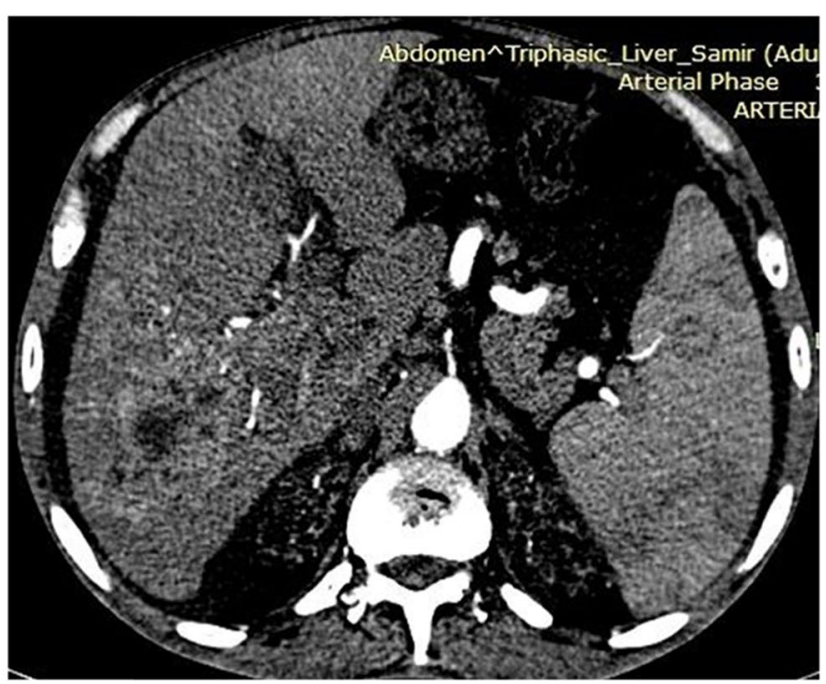

b

Fig. 2 a, b Axial CT (HAP) showing diffuse infiltrative HCC on top of the cirrhotic liver with expansion of the right and left portal veins with a thrombus that showed enhancement in HAP (tumoral thrombus)

Table 3 Correlations between s. Talin-1 and different variables

\begin{tabular}{llll}
\hline Variables & $\begin{array}{l}\text { S. Talin } \\
(\boldsymbol{N}) \text { Mean } \pm \text { SD }\end{array}$ & rs & $\boldsymbol{p}$-value \\
\hline CHILD score & & 00.051 & 0.666 \\
A & $(14) 8.90 \pm 3.76$ & & \\
B & $(40) 7.05 \pm 3.01$ & & \\
C & $(21) 8.48 \pm 2.53$ & $0.817^{*}$ & $<0.001^{*}$ \\
BCLC Staging & & & \\
A & $(10) 5.44 \pm 0.93$ & & \\
B & $(20) 7.43 \pm 1.65$ & & 0.9 \\
C & $(13) 9.36 \pm 1.59$ & & \\
$\quad$ D & $(17) 11.60 \pm 2.0$ & & \\
Sex (Male/ Female) & $(7.79 \pm 2.90)$ Vs $(7.81 \pm 3.96)$ & & \\
Vascular invasion & & & \\
$\quad$ No & $(37) 7.72 \pm 2.63$ & & \\
$\quad$ Yes & $(23) 10.27 \pm 2.12$ & $0.031^{*}$ \\
Tumor size (rs) & & $0.267^{*}$ & $0.040^{*}$ \\
\hline
\end{tabular}

rs Spearman coefficient, ${ }^{*} p$ value significant at $p \leq 0.05$

Talin-1 was significantly higher in patients with HCC compared with cirrhotic patients and healthy subjects. Thus, serum TALIN 1 has clear potential as a diagnostic tumor marker for HCC, especially in HCC patients with normal AFP values, and can be used effectively to distinguish HCC patients from healthy and cirrhotic subjects. More or less similar studies were conducted by Youns et al. [16] and Aboelfotoh et al. [17]. On another hand, contradictory results had been demonstrated by Mashaly et al. [18] that serum talin-1 in HCC patients was significantly lower than that in patients with cirrhosis and normal control.

We also observed a significant positive correlation between the level of Talin-1 and the degree of tumor burden of HCC. These findings suggest that the measurement of serum levels of Talin-1 may improve earlier detection of HCC and could potentially be a useful novel prognostic marker.

Regarding the correlation between serum Talin-1 level and size of the tumor, the present study revealed that there was a significant correlation between serum Talin-1 level and tumor size $(r=0.267, p=0.040)$. Similar observations were reported by Fang et al. [19] who confirmed that Talin-1 knockdown MHCC-97 L cells when inoculated in animals, smaller tumors were obtained in comparison with those of the control groups. So that downregulation of Talin-1 is correlated with small tumor size. On the contrary, Aboelfotoh et al. [17] found no significant correlation was detected with tumor size.

In the present study, there was a significant correlation between serum Talin-1 level and the presence of metastatic tumors and vascular invasion. Similar observations were reported by Kanamori et al. [13] who studied the correlation of overexpression of Talin-1 and HCC metastasis by differential tissue proteome as a novel molecular marker for HCC metastasis and had revealed a correlation between Talin-1 upregulation and a shorter time of recurrence after resection, which may be related to the higher rate of portal vein invasion in HCC. This shows that Talin-1 may serve as a prognostic marker for HCC. Another finding that Talin-1 overexpression is strongly 
linked to a more aggressive form of HCC suggests that Talin-1 may play an important role in the metastasis of HCC. Also, our results go hand in hand with the findings of Fang et al. [20] who found that elevated Talin-1 expression was correlated with increased invasion and migration. Moreover, Sakamoto et al. [21] reported that the overexpression of Talin-1 enhanced prostate cancer cell adhesion, migration, and invasion by activating FAK/ AKT signaling (survival signals). So, it is attempting to propose a potential strategy to block Talin-1-mediated survival signaling in tumor cells. A more or less similar study was conducted by Fang et al. [19] who used the interference technology to knockdown Talin-1 expression in MHCC-97 L cells, reported that the Talin-1 knockdown MHCC-97 L cells showed reduced Talin-1 mRNA and protein expressions, as well as arrested cell cycle in the G0/G1 phase, decreased invasion and migration abilities, confirming the involvement of Talin-1in HCC progression.

As regards the correlation between Talin-1 and tumor metastasis, contradictory results were reported by Zhang et al. [11] who found that the expression of Talin-1 protein and mRNA in HCC tissues was significantly lower than that in the adjacent non-cancerous tissues and normal liver tissues by immunohistochemistry and real-time PCR [11]. Also, Mashaly et al. [18] mentioned that increased invasion and metastasis correlated with reduced Talin-1 level. These results may be explained by the loss of Talin in the tumor tissue indicates a significant change in the integrity of these cancer tissues, and the possibility that individual cells may break away from the parent histology due to loss of cell adhesion, ultimately leading to tumor angiogenesis and development. Aravalli et al. [22] explained that Talin-1 exerts different effects in the formation of different cancer, which are the multistep and complex processes associated with the accumulation of genetic and epigenetic changes.

\section{Limitations}

One of the limitations of this study was small sample size and narrow cut-off value for diagnosis and differentiation of HCC between the studied groups, so further studies should be replicated at a multicentric study level with large sample size. Also, follow-up of serum Talin levels after treatment of HCC by the available different treatment modalities could add to the strength of its diagnostic and prognostic performance as a new HCC tumor marker.

\section{Conclusion}

In conclusion, the present study has shown that serum Talin-1 is significantly increased in HCC patients and has significantly different serum levels among HCC patients in comparison to cirrhotic patients and controls. Talin-1 is significantly related to tumor progression in HCC patients as it is closely related to the BCLC staging system, portal vein invasion, and tumor size. Therefore Talin-1 may be considered as a useful biomarker for diagnosis as well as predicting metastatic potential and vascular invasion of HCC. Serum Talin-1 could be added to AFP as a serological biomarker with higher sensitivity in the detection of HCC. The usefulness of serum Talin-1 testing alone for the population at risk should be further investigated on considerably large sample size to validate its use.

\section{Acknowledgements}

We are indebted to nursing staff at Hepatobiliary Unit and outpatients' clinic of Alexandria Main University Hospital, Alexandria, Egypt, for helping in collecting serum samples.

\section{Authors' contributions}

$\mathrm{El}$, designed, conceived the idea of the study, and supervised the study. HN, collection of data, prepared the scientific contents, and drafted the article. $\mathrm{DM}$, interpretation of radiological findings and reviewing of the article. ET, interpretation of data, serum sample analysis, and reviewing of the article. MT, participated in designing the study methodology, shared in data collection, supervised the study, and drafted the article. The authors reviewed and approved the final manuscript.

\section{Funding}

None.

\section{Availability of data and materials}

Available on request from any author.

\section{Declarations}

\section{Ethics approval and consent to participate}

The study protocol was approved by the research review committee of the Alexandria Faculty of Medicine and was conformed to the 1975 Declaration of Helsinki. Informed consent was obtained from each subject included in the study.

\section{Consent for publication}

The authors approved the publication process conducted by the corresponding author.

\section{Competing interests}

The authors declare that they have no competing interests.

\section{Author details}

${ }^{1}$ Hepatobiliary Unit, Internal Medicine Department, Alexandria Faculty of Medicine, Alexandria University, Alexandria, Egypt. ${ }^{2}$ Department of Radiology, Alexandria Faculty of Medicine, Alexandria University, Alexandria, Egypt. ${ }^{3} \mathrm{Clinical}$ Pathology Department, Alexandria Faculty of Medicine, Alexandria University, Alexandria, Egypt. ${ }^{4}$ Clinical Science Department, College of Medicine, Princess Nourah bint Abdulrahman University, Riyadh, KSA.

Received: 4 November 2021 Accepted: 21 February 2022

Published online: 28 February 2022

\section{References}

1. Kokudo N, Hasegawa K, Akahane M, Igaki H, Izumi N, Ichida T et al (2015) Evidence-based clinical practice guidelines for hepatocellular carcinoma: 
the Japan Society of Hepatology 2013 update (3rd JSH-HCC Guidelines). Hepatol Res 45(2):123-127

2. Addario L, Tritto G, Cavaglia E, Amodio F, Giannelli E, Di Costanzo GG (2011) Preserved liver function, portal thrombosis and absence of oesophageal varices are risk factors for metastasis of hepatocellular carcinoma. Dig Liver Dis 43(4):319-324

3. El-Zayadi AR, Badran HM, Shawky S, Emara S, El-Bareedy A, Sobhi M (2010) Effect of surveillance for hepatocellular carcinoma on tumor staging and treatment decisions in Egyptian patients. Hepatol Int 4(2):500-506

4. American Cancer Society (2015) Cancer facts and figures 2015. American Cancer Society, Atlanta

5. Center MM, Jemal A (2011) International trends in liver cancer incidence rates. Cancer Epidemiol Biomarkers Prev 20(11):2362-2368

6. Anwar WA, Khaled HM, Amra HA, El-Nezami H, Loffredo CA (2008) Changing pattern of hepatocellular carcinoma (HCC) and its risk factors in Egypt: possibilities for prevention. Mutat Res 659(1-2):176-184

7. European Association For The Study OfThe Liver, European Organisation For Research Treatment Of Cancer (2012) EASL-EORTC clinical practice guidelines: management of hepatocellular carcinoma. J Hepatol 56(4):908-943

8. Daniele B, Bencivenga A, Megna AS, Tinessa V (2004) Alpha-fetoprotein and ultrasonography screening for hepatocellular carcinoma. Gastroenterology 127:108-112

9. Teofanescu I, Gologan E, Stefanescu G, Balan G (2010) Surveillance of cirrhosis for hepatocellular carcinoma--clinical validation of new serological biomarkers for improved diagnosis. Rev Med Chir Soc Med Nat lasi 114(1):39-46

10. Goult BT, Gingras AR, Bate N, Barsukov IL, Critchley DR, Roberts GC (2010) The domain structure of talin: residues 1815-1973 form a five-helix bundle containing a cryptic vinculin-binding site. FEBS Lett 584(11):2237-2241

11. Zhang JL, Qian YB, Zhu LX, Xiong QR (2011) Talin1, a valuable marker for diagnosis and prognostic assessment of human hepatocelluar carcinomas. Asian Pac J Cancer Prev 12(12):3265-3269

12. Sakamoto S, McCann RO, Dhir R, Kyprianou N (2010) Talin1 promotes tumor invasion and metastasis via focal adhesion signaling and anoikis resistance. Cancer Res 70(5):1885-1895

13. Kanamori $\mathrm{H}$, Kawakami T, Effendi K, Yamazaki K, Mori T, Ebinuma $\mathrm{H}$ et al (2011) Identification by differential tissue proteome analysis of talin-1 as a novel molecular marker of progression of hepatocellular carcinoma. Oncology. 80(5-6):406-415

14. Kopp PM, Bate N, Hansen TM, Brindle NP, Praekelt U, Debrand E et al (2010) Studies on the morphology and spreading of human endothelial cells define key inter- and intramolecular interactions for talin1. Eur J Cell Biol 89(9):661-673

15. Cabrera R, Fitian Al, Ararat M, Xu Y, Brusko T, Wasserfall C et al (2012) Serum levels of soluble CD25 as a marker for hepatocellular carcinoma. Oncol Lett 4(4):840-846

16. Youns MM, Abdel Wahab AH, Hassan ZA, Attia MS (2013) Serum talin-1 is a potential novel biomarker for diagnosis of hepatocellular carcinoma in Egyptian patients. Asian Pac J Cancer Prev 14(6):3819-3823

17. Aboelfotoh AO, Foda EM, Elghandour AM, Teama NM, Abouzein RA, Mohamed GA (2020) Talin-1; other than a potential marker for hepatocellular carcinoma diagnosis. Arab J Gastroenterol 21:80-84

18. Mashaly AH, Anwar R, Ebrahim MA, Eissa LA, El Shishtawy MM (2018) Diagnostic and prognostic value of Talin-1 and midkine as tumor markers in hepatocellular carcinoma in Egyptian patients. Asian Pac J Cancer Prev 19:1503-1508

19. Fang KP, Dai W, Ren YH, Xu YC, Zhang SM, Qian YB (2016) Both Talin-1 and Talin-2 correlate with malignancy potential of the human hepatocellular carcinoma MHCC-97 L cell. BMC Cancer. 16:45

20. Fang KP, Zhang JL, Ren YH, Qian YB (2014) Talin-1 correlates with reduced invasion and migration in human hepatocellular carcinoma cells. Asian Pac J Cancer Prev 15(6):2655-2661

21. Sakamoto S, Kyprianou N (2010) Targeting anoikis resistance in prostate cancer metastasis. Mol Aspects Med 31(2):205-214

22. Aravalli RN, Steer CJ, Cressman EN (2008) Molecular mechanisms of hepatocellular carcinoma. Hepatology. 48(6):2047-2063

\section{Publisher's Note}

Springer Nature remains neutral with regard to jurisdictional claims in published maps and institutional affiliations.

\section{Submit your manuscript to a SpringerOpen ${ }^{\circ}$ journal and benefit from:}

- Convenient online submission

- Rigorous peer review

- Open access: articles freely available online

- High visibility within the field

- Retaining the copyright to your article

Submit your next manuscript at $\boldsymbol{\nabla}$ springeropen.com 